\title{
MLH1 enhances the sensitivity of human endometrial carcinoma cells to cisplatin by activating the MLH1/c-Abl apoptosis signaling pathway
}

\author{
Yue Li ${ }^{1,2}$, Shihong Zhang ${ }^{2}$, Yuanjian Wang $^{3}$, Jin Peng ${ }^{1}$, Fang Fang ${ }^{2}$ and Xingsheng Yang ${ }^{1 *}$ (D)
}

\begin{abstract}
Background: MLH1 plays a critical role in maintaining the fidelity of DNA replication, and defects in human MLH1 have been reported. However, the role of MLH1 in endometrial carcinoma has not been fully investigated. Therefore, we aimed to study the role of MLH1 in the sensitivity of human endometrial carcinoma cells to cisplatin.

Methods: In this study, we detected the expression of MLH1 in Ishikawa and RL95-2 cells. MLH1-siRNA and ADV-MLH1 were adopted for the silencing and overexpression of $\mathrm{MLH1}$, respectively. Real-time polymerase chain reaction, Western blotting, cell proliferation assays, and cell cycle and apoptotic analyses by flow cytometry were employed to explore the underlying mechanism. A mouse xenograft model was used to investigate the effect of MLH1 on tumor growth after treatment with cisplatin.

Results: Over-expression of MLH1 in Ishikawa cells dramatically increased the sensitivity of cells to cisplatin and enhanced cell apoptosis. By contrast, knockdown of MLH1 yielded the opposite effects in vitro. Mechanistically, cisplatin induced the MLH1/c-Abl apoptosis signaling pathway in ADV-MLH1-infected endometrial carcinoma cells, and these effects involved c-Abl, caspase-9, caspase-3 and PARP. Altogether, our results indicate that ADVMLH1 might attenuate Ishikawa cell growth in vivo, resulting in increased cisplatin sensitivity.

Conclusions: MLH1 may render endometrial carcinoma cells more sensitive to cisplatin by activating the MLH1/ c-Abl apoptosis signaling pathway. In addition, an applicable adenovirus vector (ADV-MLH1) for MLH1 overexpression in endometrial carcinoma was generated. Thus, ADV-MLH1 might be a novel potential therapeutic target for endometrial carcinoma.
\end{abstract}

Keywords: Endometrial carcinoma, siRNA-MLH1, ADV-MLH1, Cisplatin sensitivity, Individualized therapy

\section{Background}

Endometrial carcinoma is a common malignant tumor in the female reproductive system. Women with hereditary nonpolyposis colorectal cancer exhibit a 40 to $60 \%$ cumulative lifetime risk for endometrial carcinoma, which arises as a result of a genetic predisposition to the disease, characterized by mutations in DNA mismatch repair (MMR) genes such as MLH1 and MSH2 [1]. Defects in

\footnotetext{
* Correspondence: xingshengyang@sdu.edu.cn

'Department of Obstetrics and Gynecology, Qilu Hospital of Shandong University, No. 107, Wenhua Xi Road, Jinan, Shandong 250012, People's Republic of China

Full list of author information is available at the end of the article
}

MMR proteins give rise to genome instability, which is a characteristic of most cancers, especially hereditary cancers $[2,3]$. Loss of DNA mismatch repair caused by MMR deficiency also accounts for the cytotoxicity induced by specific types of DNA-damaging chemotherapeutic agents (e.g., alkylating agents and cisplatin) $[4,5]$. Thus, MMR is essential for effective cancer therapy and individual health.

Various models have demonstrated drug resistance caused by low levels of the MLH1 protein in ovarian and esophageal tumor samples following cisplatin (cis-dichlorodiammine platinum, CDDP)-based chemotherapy. Additionally, several studies, which examined MMR protein levels and microsatellite instability in germ cell

(C) The Author(s). 2018 Open Access This article is distributed under the terms of the Creative Commons Attribution 4.0 International License (http://creativecommons.org/licenses/by/4.0/), which permits unrestricted use, distribution, and 
tumors from patients receiving cisplatin-based chemotherapy, have shown the prognostic value of prechemotherapy MMR protein status in these tumors $[6,7]$. Sawant et al. demonstrated that loss of base excision repair and MMR proteins gives rise to cisplatin resistance, and these two pathways share the same mechanism in mediating cisplatin sensitivity $[8,9]$. It has also been observed that decreased cellular cytotoxicity is induced by increased repair of cisplatin interstrand crosslinks in the absence of MMR proteins [10]. The potential relevance of these findings underscores the need for a greater understanding of the role of MLH1 in mediating cisplatin sensitivity. In this study, we investigated the role of MLH1 in the sensitivity of human endometrial carcinoma cells to cisplatin and generated an adenovirus vector (ADV) ADV-MLH1 that can be widely applied for selective overexpression of MLH1, which represents a potential therapeutic target for endometrial carcinoma. No similar research has been reported internationally.

\section{Methods \\ Cell culture}

Ishikawa and RL95-2 cells were generously donated by the Gynecologic Oncology Laboratory at Qilu Hospital in Shandong Province, China. RL95-2 cells were maintained in Dulbecco's modified Eagle's medium/F-12 media (HyClone, Biological Industries, Israel) with $10 \%$ fetal bovine serum (FBS, Invitrogen, USA) with antibiotics, whereas Ishikawa cells were maintained in Roswell Park Memorial Institute (RPMI) modified medium (HyClone, Biological Industries, Israel) supplemented with 10\% FBS (Invitrogen, USA) with antibiotics. All cell lines were cultured in a humidified atmosphere of $5 \% \mathrm{CO}_{2}$ at $37^{\circ} \mathrm{C}$. Half of the medium was replaced with fresh medium at 3-day intervals until the attached cells reached $70-80 \%$ confluence in our experiments.

All experimental procedures were approved by the Laboratory Animal Ethics Committee of Qilu Hospital, Shandong University. The principles outlined in the ARRIVE (Animal Research: Reporting of In Vivo Experiments) guidelines and the Basel declaration (including the $3 \mathrm{R}$ concept) were considered when planning experiments.

\section{Reagents and antibodies}

Cisplatin was purchased from Sigma-Aldrich (USA), dissolved in dimethyl sulfoxide (DMSO, Solarbio, Beijing, China) to a stock concentration of $10 \mathrm{mM}$, and stored in single-use aliquots at $-80^{\circ} \mathrm{C}$. An anti-MLH1 antibody was purchased from Abcam (ab92312, United Kingdom). Anti-p-c-Abl, anti-cleaved caspase-3, anti-cleaved caspase9, and anti-cleaved PARP antibodies were purchased from Cell Signaling Technology Inc. (China). Anti-BCL-2 antibody was purchased from Proteintech Group Inc. (USA).
An anti- $\beta$-actin antibody was purchased from Zhongshan Jinqiao biotechnology Co., Ltd. (Beijing, China).

\section{Real-time polymerase chain reaction (PCR) for measurement of MLH1 transcript levels}

Total RNA was isolated using TRIzol (Invitrogen, USA) according to the manufacturer's instructions. First-strand cDNA synthesis was performed using the Moloney murine leukemia virus (M-MLV) reverse transcriptase enzyme (Invitrogen, USA) according to the manufacturer's protocol. Transcript levels were quantified using SYBR Premix Ex Taq (Takara Bio Inc., Japan) in an Applied Biosystems StepOne Plus Real-time PCR System. Detailed methods are available for download at www.takara-bio.com. U6 served as an endogenous control. The fold-changes in mRNA expression levels were determined using the $2^{-\triangle \Delta C T}$ method $[11,12]$. Primers were designed by Genepharma Company (Shanghai, China). The primer sequences are presented in Additional file 4: Table S1.

\section{Western blot analysis}

Proteins were extracted after treatment using a standard method, and protein concentrations were determined using the Bradford method according to Kruger [13]. Proteins were extracted with RIPA buffer $(50 \mathrm{mM}$ Tris $\mathrm{pH}$ 8.0, $150 \mathrm{mM} \mathrm{NaCl}, 1 \% \mathrm{NP}-40,1 \mathrm{mM}$ ethylenediaminetetraacetic acid (EDTA), and $0.1 \%$ sodium dodecyl sulfate (SDS) containing protease inhibitors). Total protein $(30 \mu \mathrm{g})$ was separated in a $12 \%$ SDS gel and transferred to Immobilon-P transfer membranes (Merck Millipore, Ltd. Tullagreen, Carrigtwohill, Co. Cork IRL Rev. Size: $0.2 \mu \mathrm{m})$ after electrophoresis. After blocking with $5 \%$ nonfat dry milk, the membranes were probed with primary antibodies recognizing human MLH1 (1:1000), p-c-Abl (1:1000), cleaved caspase-3 (1:800), cleaved caspase-9 (1:500), cleaved PARP (1:500), and Bcl-2 (1:1000), followed by a horseradish peroxidaselinked secondary antibody (goat anti-rabbit IgG, 1:1000). $\beta$-actin (1:1000) served as an endogenous control. Immunoreactive proteins were visualized using the Immobilon Western Chemiluminescent substrate (ECL; Millipore Corporation, Billerica, MA, USA). The density of all proteins relative to $\beta$-actin was quantified with ImageJ (National Institutes of Health, USA). The experiment was repeated in triplicate.

\section{MLH1 overexpression and silencing}

The MLH1-pAD-kan adenovirus vector (ADV-MLH1) was utilized to upregulate MLH1 (Additional file 1: Figure S1, Additional file 2: Figure S2 and Additional file 3: Figure S3). ADV-NC served as a negative control (Additional file 4: Table S1). Cells were plated in 6-well plates and infected at 30 to $40 \%$ confluence using ADV-MLH1 ( $1 \times$ $10^{12} \mathrm{vp} / \mathrm{ml}$, Multiplicity of infection $\left.(\mathrm{MOI})=200\right)$ mixed 
with serum-free medium. The medium was changed after $12 \mathrm{~h}$. After treatment, cells were harvested at different time points and prepared for future use. We selected the optimal MLH1 siRNA fragment to silence MLH1. Cells were transfected with the indicated MLH1-siRNA (MLH1-homo-528/1832/2431) (synthesized by Genepharma, Shanghai) using the EndofectinMAX Transfection Reagent (Genecopoeia, Rockville, USA) according to the manufacturer's instructions. The sequences of MLH1-homo-528, MLH1-homo-1832 and MLH1-homo-2431 are presented in Additional file 4: Table S1. Briefly, the cells were plated in 6-well plates. At the time of transfection, cell density was maintained at 50 to $60 \%$, and the medium was changed to complete medium at 6 -h intervals. The cells were harvested at different time points for the detection of expression levels.

\section{Cell proliferation assays}

An enhanced cell counting kit-8 (CCK-8, Beyotime, Beijing) assay was utilized to determine cell proliferation as previously described [14] ' Cells were plated in 96-well plates at a density of 8000 to 10,000 cells/well. Viable cell absorbance values were determined using a NanoQuant Infinite M200 PRP (TECAN, Austria) at $490 \mathrm{~nm}$, and the data were analyzed with SoftMax software. Cell viability was expressed by normalizing untreated cells to $100 \%$. The experiment was performed in triplicate for each cisplatin concentration.

\section{Flow cytometry analysis of the cell cycle and apoptosis}

The cell cycle was analyzed using a Cell Cycle Detection Kit (BB-4104, BestBio, Shanghai) according to the manufacturer's instructions. The cells were harvested by centrifugation and then fixed in $75 \%$ cold ethanol overnight after washing three times with PBS. Each sample was washed and resuspended in $500 \mu \mathrm{l}$ of propidium iodide (PI) working solution for 30 to $60 \mathrm{~min}$ in the dark before detection using a FACSCalibur flow cytometer (Becton, Dickinson and Company, USA). The percentage of cells was further analyzed with ModFit LT software. Cell apoptosis was measured using an Annexin V-FITC/PI staining kit (BB-4101, BestBio, Shanghai) according to the manufacturer's guidelines. The cells were counted and adjusted to $1 \times 10^{6} \mathrm{cell} / \mathrm{ml}$. Then, a $2 \mathrm{ml}$ aliquot of the cells was inoculated into each well of a 6-well plate, and the cells were allowed to grow for $24 \mathrm{~h}$ prior to drug treatment. Flow cytometry was performed using a FACSCalibur flow cytometer (Becton, Dickinson and Company, USA) and processed using CellQuest Pro analysis software. The experimental methods used for flow-cytometric analysis in our study can be found in previous reports $[15,16]$.
Animal experiments and immunohistochemistry staining Six-week-old female BALB/c nude mice weighing 16 to $18 \mathrm{~g}$ were purchased from Vital River (Beijing, China). All animal experiments complied with the ARRIVE guidelines and were performed in accordance with the National Institutes of Health Guide for the Care and Use of Laboratory Animals (NIH Publications No. 8023, revised 1978). This experiment was designed for 6 animals per group. Briefly, $1 \times 10^{7}$ Ishikawa cells were suspended in $200 \mu \mathrm{l}$ of PBS and injected subcutaneously into the left flank of each mouse. When the tumor diameter reached 4 to $6 \mathrm{~mm}$, the mice in each group received the first intratumoral injection of ADV-MLH1 and ADV-NC (each at $2 \times 10^{9} \mathrm{VP} /$ tumor). We repeated the intratumoral injection of the same adenoviral vector $\left(2 \times 10^{9}\right.$ $\mathrm{VP} /$ tumor) at days 5, 9, 13 and 17. Cisplatin treatment was performed via intraperitoneal injection of $5 \mathrm{mg} / \mathrm{kg}$ cisplatin in $0.9 \%$ physiological saline at days $4,11,18$ and 25. Tumor volume was calculated as follows: V (tumor) $=\mathrm{D} \times\left(\mathrm{d}^{2}\right) .(\mathrm{D}=$ length, $\mathrm{d}=$ width, $\mathrm{d}<\mathrm{D})$.

MLH1 protein expression in mouse tumor tissue from each group (ADV-MLH1 + CDDP-treated mice, ADV-NC + CDDP-treated mice) was assessed by immunohistochemistry staining. Standard three-step, indirect immunohistochemical analysis was conducted in 4- $\mu \mathrm{m}$ tissue sections that had been transferred to glass slides and included the following steps: citrate antigen retrieval, endogenous peroxidase blockage and avidin-binding activity and di-aminobenzidine development [17]. Primary antibody against MLH1 (dilution, 1:200) (Abcam, ab92312, United Kingdom) was used, and the corresponding secondary antibodies were goat anti-rabbit IgG antibodies (cat. no. SP-9000; Zhongshan Jinqiao biotechnology Co., Ltd., Beijing, China). Staining patterns were analyzed by at least two experienced pathologists affiliated with the Department of Pathology of Qilu Hospital of Shandong University.

\section{Statistical analysis}

All statistical analyses were performed in GraphPad Prism version 6.01. Differences between two or three groups were analyzed using Student's t-test. $P$-values < 0.05 were considered statistically significant.

\section{Results}

MLH1 protein and mRNA levels in endometrial carcinoma cells subjected to different treatments

To study the role of MLH1 in endometrial carcinoma, we first confirmed the protein and mRNA levels of MLH1 in two human endometrial cancer cell lines (Ishikawa and RL95-2 cells) and investigated the overexpression and silencing of MLH1 in these cells. As shown in Fig. 1a, lower MLH1 expression was detected in Ishikawa cells than in RL95-2 cells $(3.9 \%$ VS $79.9 \%$; $P<$ 


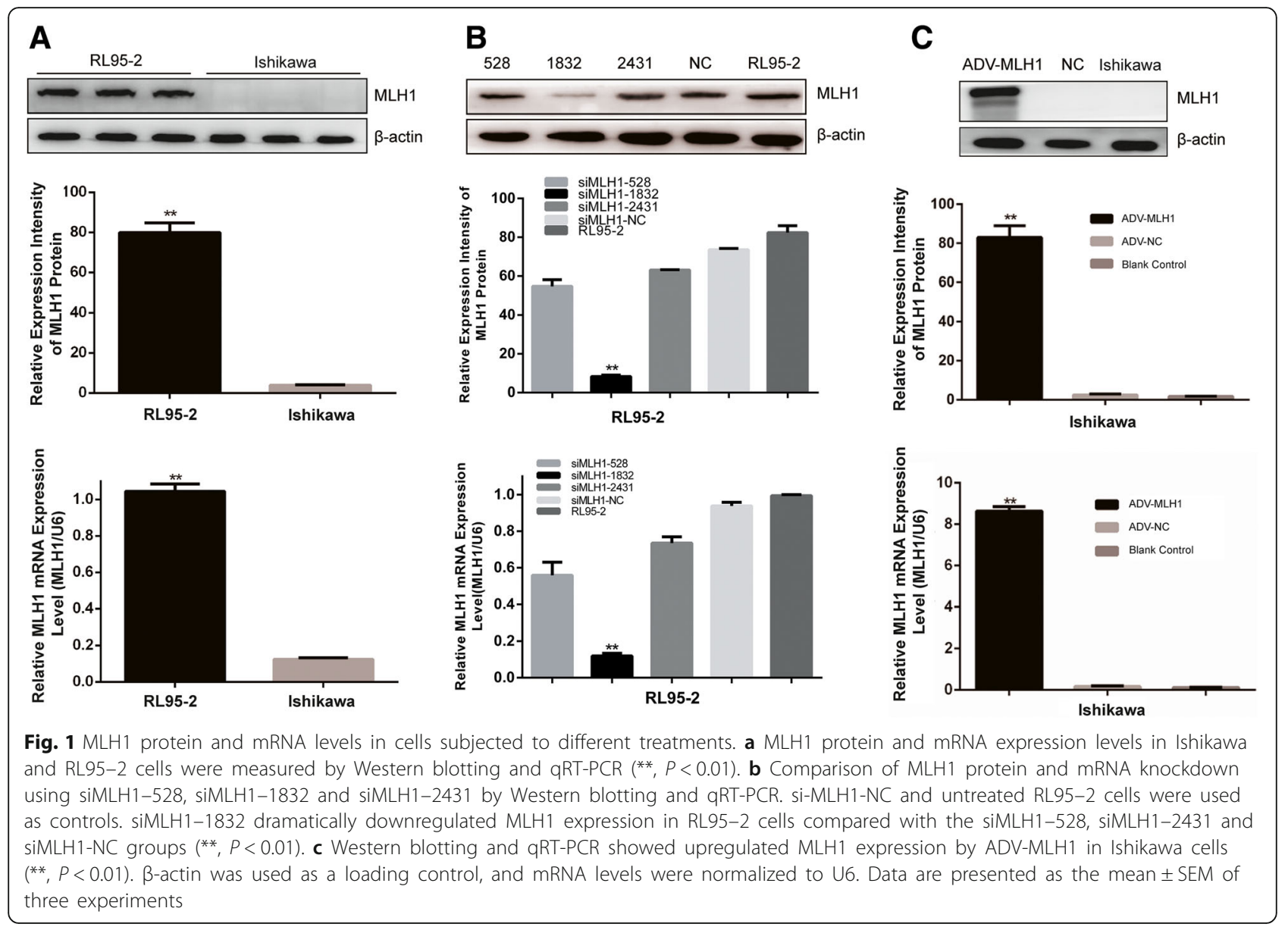

0.01). Next, we analyzed MLH1 expression levels in cells subjected to different treatments. siMLH1-1832 reduced MLH1 protein levels by more than 90\% in RL95-2 cells compared with siMLH1-528, siMLH1-243 and negative control siMLH1-NC. MLH1 silencing was confirmed by Western blotting and quantitative RT-PCR (qRT-PCR; $P$ $<0.01$; Fig. 1b). Additionally, we generated an adenovirus vector (ADV-MLH1) for overexpressing MLH1. Elevated MLH1 protein and mRNA levels in Ishikawa cells were confirmed by Western blotting and qRT-PCR compared with the controls $(* *, P<0.01$; Fig. 1c).

\section{MLH1 affected the sensitivity and cell cycle of endometrial carcinoma cells in response to cisplatin in vitro}

Sensitization to some chemotherapeutic agents is thought to be associated with functional MMR [18, 19]. The different MLH1 expression levels in endometrial carcinoma cells prompted us to examine whether overexpression or knockdown of MLH1 affects cell proliferation in response to cisplatin. Figure 2a presents the results of CCK-8 assays in which MLH1-proficient RL95-2 cells and MLH1-deficient Ishikawa cells were treated with different concentrations of cisplatin (0-
$25 \mu \mathrm{mol}$ ) for 24,48 and $72 \mathrm{~h}$. The figure shows that the viability of RL95-2 (MLH1-proficient) cells was considerably reduced compared with that of Ishikawa (MLH1deficient) cells upon exposure to cisplatin, revealing concentration- and time-dependent suppression (Fig. 2a). Cell viability was significantly increased in siMLH1-transfected RL95-2 cells compared with control cells (Fig. 2b). We also detected significantly decreased tolerance to CDDP in ADV-MLH1-infected Ishikawa cells compared with controls (Fig. 2c). Meanwhile, DMSO (CDDP solvent) alone had no effect on cell viability (data not shown). These results indicated that re-expressing MLH1 increased the sensitivity of Ishikawa cells to cisplatin, and silencing MLH1 decreased RL95-2 cell sensitivity to cisplatin. As illustrated in Fig. $2 \mathrm{~d}$ and e, a slight increase in the number of cells in G1/G0 $(P<0.05)$ was observed in ADV-MLH1-infected Ishikawa cells, whereas a decrease in the distribution of the G1/G0 peak $(P<0.01)$ and an increase in the percentage of $S$-phase cells $(P<0.01)$ were observed in MLH1-downregulated cells (RL95-2) compared with the NC group. Altogether, these results indicate that MLH1 affects the sensitivity and cell cycle of endometrial carcinoma cells in response to cisplatin in vitro. 


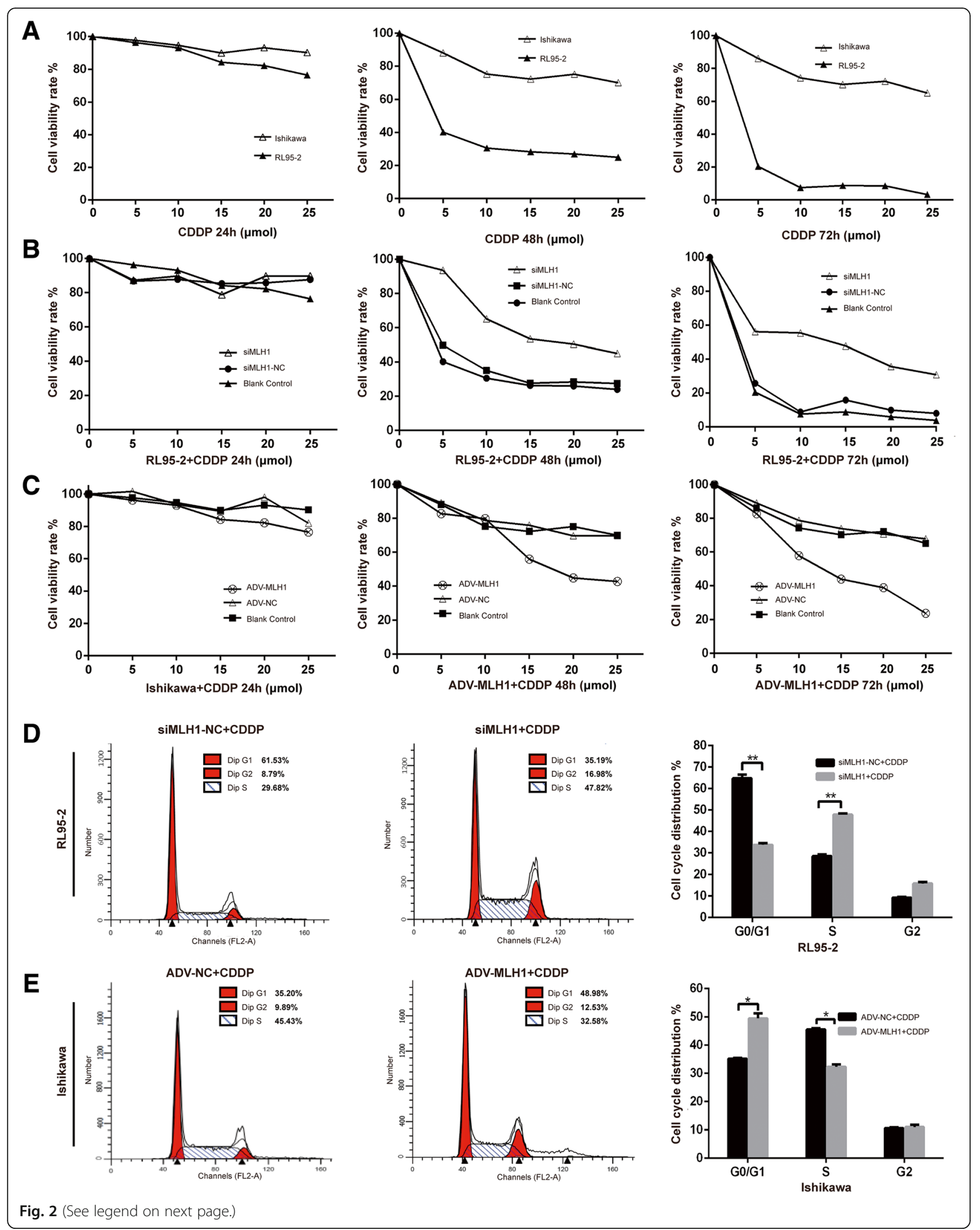


(See figure on previous page.)

Fig. $2 \mathrm{MLH1}$ affected endometrial carcinoma cell proliferation in response to cisplatin in vitro. a RL95-2 and Ishikawa cell growth curves after 24 $\mathrm{h}, 48 \mathrm{~h}$ and $72 \mathrm{~h}$ of treatment with different concentrations of cisplatin ( $0-25 \mu \mathrm{mol})$. b Viability rates of RL95-2 cells after MLH1 silencing. Cells transfected with siMLH1-NC and normal RL95-2 cells served as controls. $\mathbf{c}$ Viability rates of Ishikawa cells after MLH1 overexpression. The viability rate of ADV-MLH1/ADV-NC-infected Ishikawa cells was normalized to control cells. Each value represents the mean of at least three independent experiments performed in triplicate (CCK-8). d Distribution of the cell cycle in response to $5 \mu \mathrm{mol}$ cisplatin in MLH1 knockdown RL95-2 cells compared with the control. e Distribution of the cell cycle in response to $25 \mu \mathrm{mol}$ cisplatin in MLH1-overexpressing cells compared with the control

MLH1 might enhance the cisplatin-induced apoptosis of human endometrial carcinoma cells in vitro

To further characterize the important role of MLH1 in improving the chemosensitivity of human endometrial carcinoma, we assessed the effect of MLH1 on cisplatininduced apoptosis using flow cytometry. For apoptotic analysis, as illustrated in Fig. $3 a$ and b, the distribution of apoptotic cells was analyzed $72 \mathrm{~h}$ after cisplatin treatment. Compared with cells treated with siMLH1/ siMLH1-NC, ADV-MLH1/ADV-NC or cisplatin alone, the apoptotic and early apoptotic fractions were increased by 1.51 -fold $(P<0.05)$ in ADV-MLH1-infected Ishikawa cells and decreased by 3.54 -fold $(P<0.01)$ in siMLH1-transfected RL95-2 cells.

\section{Cisplatin might activate the MLH1/c-Abl apoptosis} signaling pathway in endometrial carcinoma cells Li et al. suggested that c-Abl mediates MLH1-dependent apoptosis in colon cancer cells [20]. Therefore, we examined the potential participation of c-Abl, B-cell lymphoma2 (bcl-2), caspase-9, caspase- 3 and PARP in the cisplatin- induced MLH1/c-Abl apoptosis signaling pathway in endometrial carcinoma cells via Western blot analysis. The phosphorylation of the $\mathrm{c}$-Abl protein was upregulated in Ishikawa cells overexpressing MLH1, demonstrating MLH1 dependence beginning at $48 \mathrm{~h}$ and increasing until $72 \mathrm{~h}$ after treatment with cisplatin. Quantification of phosphorylated c-Abl processing by densitometry revealed that MLH1-overexpressing Ishikawa cells displayed 1.6- and 2.0-fold increases in c-Abl phosphorylation at 48 and $72 \mathrm{~h}$, respectively, compared with control cells $(P<0.01$; Fig. 4 a). Similar trends were observed for cleaved caspase-9, cleaved caspase- 3 and cleaved PARP. Cleaved caspase- 3 activity was increased 2.3- and 2.5-fold after the administration of ADV-MLH1 in Ishikawa cells for 48 and 72 h $(P<0.01)$. Compared with control cells, cleaved PARP was increased 1.68 -fold at $48 \mathrm{~h}(*, P<0.05)$ and 2.2 -fold at $72 \mathrm{~h}(P<0.01)$ (Fig. 4a). However, when MLH1 expression was downregulated, opposing trends in the levels of these proteins were observed compared with control cells (Fig. 4b).

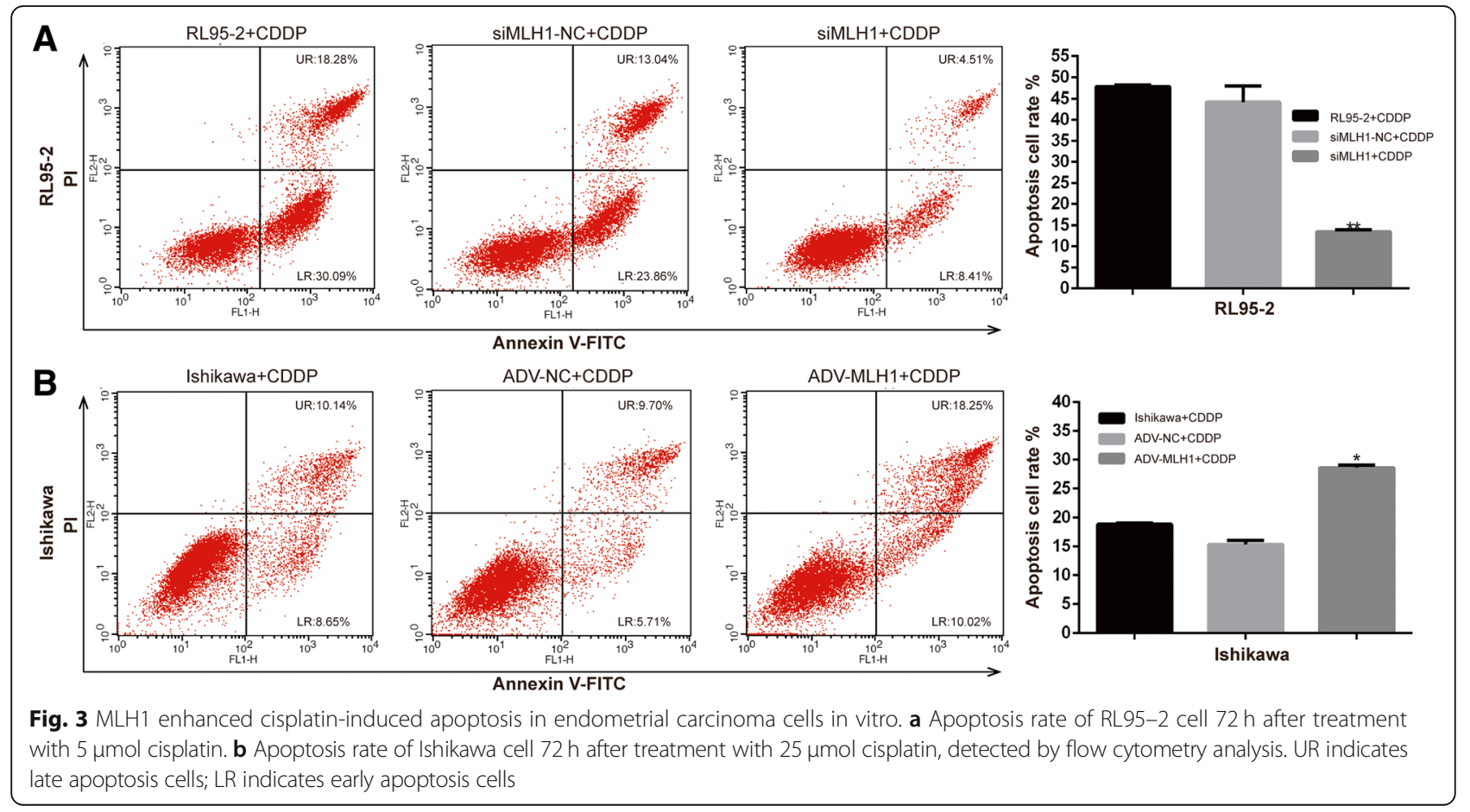




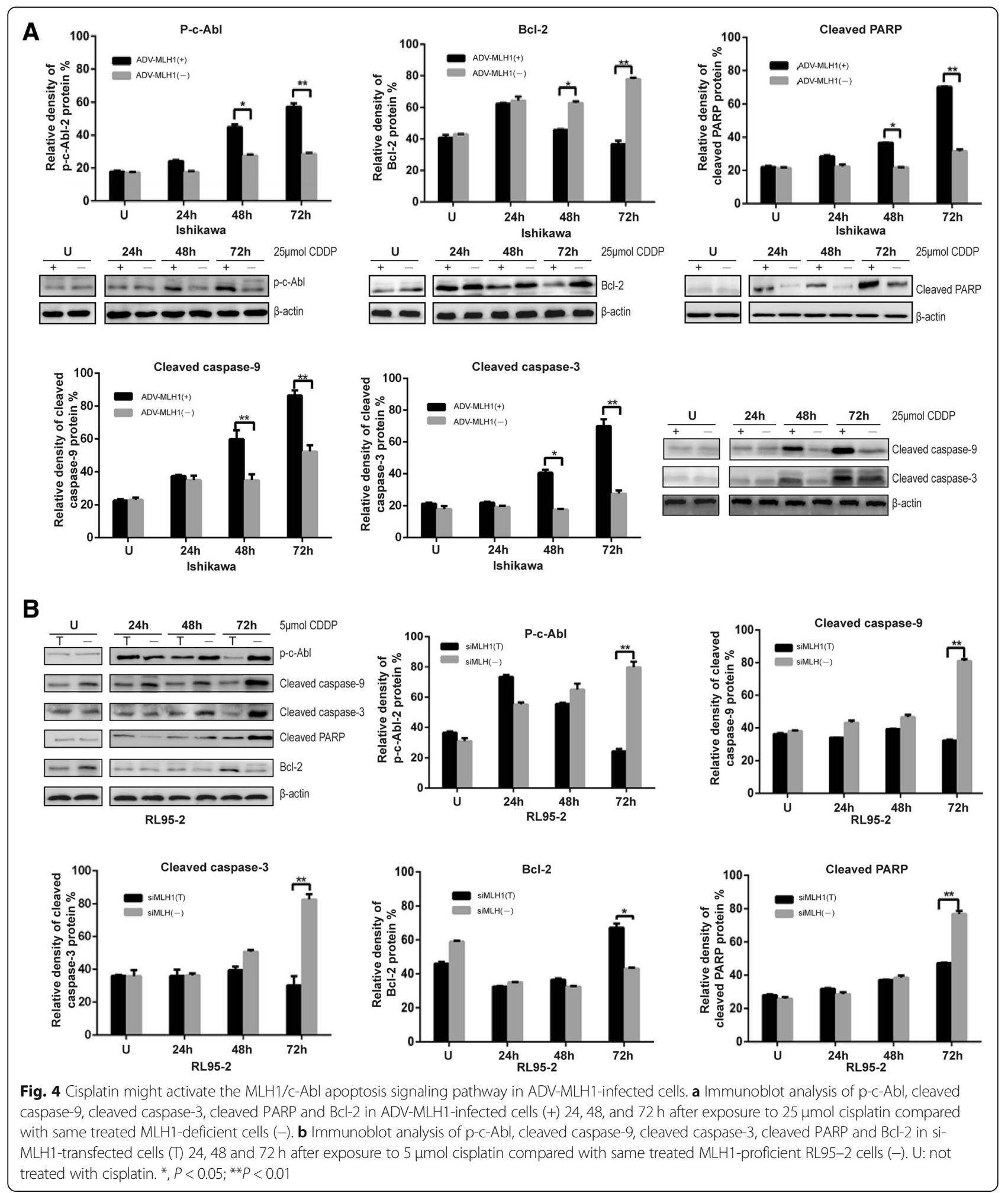

On the other hand, the expression of the Bcl-2 protein gradually decreased in a time-dependent manner in MLH1-re-expressing Ishikawa cells from $48 \mathrm{~h}$ to $72 \mathrm{~h}$ after cisplatin treatment (Fig. 4a). When MLH1 expression was downregulated, $\mathrm{Bcl}-2$ exhibited the opposite trend (Fig. 4b). 
These results demonstrated that cisplatin might activate the $\mathrm{MLH} 1 / \mathrm{c}-\mathrm{Abl}$ apoptosis signaling pathway in endometrial carcinoma cells.

\section{Ishikawa cells infected with ADV-MLH1 display markedly attenuated tumor growth in vivo}

We subsequently investigated the effects of MLH1 on tumor growth in response to cisplatin in a mouse xenograft model. The results revealed that the tumors in the ADV-MLH1 group were much smaller than those in the ADV-NC group. On days 25 and 30, mice in the ADVMLH1 + CDDP group exhibited a significantly reduced tumor size compared with those in the ADV-NC control group $(P<0.01$, Fig. $5 a)$. On day 30 , tumor size in the ADV-NC group increased to an average of $603.0 \mathrm{~mm}^{3}$, compared with $207.6 \mathrm{~mm}^{3}$ in the ADV-MLH1 group ( $P$ $<0.05$ ) (Fig. 5b, c). The difference in tumor size between the ADV-MLH1 and ADV-NC control groups was significant. In addition, we performed MLH1 immunohistochemical staining in mouse tumor tissue from each group (ADV-MLH1 + CDDP-treated mice, ADV-NC + CDDP-treated mice) and observed that the ADV-MLH1 + CDDP-treated mouse tumor tissue was MLH1 positive, while the ADV-NC + CDDP-treated mouse group was
MLH1 negative (Fig. 5d). These data helped us assess the transduction efficiency in vivo, and the results indicated that ADV-MLH1 might render endometrial carcinoma cells more sensitive to cisplatin and attenuate endometrial tumor growth in vivo.

\section{Discussion}

The human MMR system plays a critical role in maintaining the fidelity of DNA replication. Mismatch recognition is performed by MutS $\alpha$, which is composed of MSH2 and MSH6, and MutS $\beta$ (MSH2-MSH3), whereas the recruitment of downstream MMR proteins is executed by MutLa, which is composed of MLH1-PMS2 [21]. MLH1, which can recognize and repair base-base mismatches and insertion/deletion loops that occur during DNA replication, is the key component of the MMR system. MLH1 also plays an important role in cell apoptosis stimulated by DNA damage [22]. MLH1deficient cells exhibit resistance when treated with DNAdamaging chemotherapeutic agents [23, 24]. Thus, MLH1 is crucial for maintaining gene integrity in cells and disease prevention. Our study is the first to demonstrate the involvement of MLH1 in cisplatin sensitivity in human endometrial carcinoma cells.

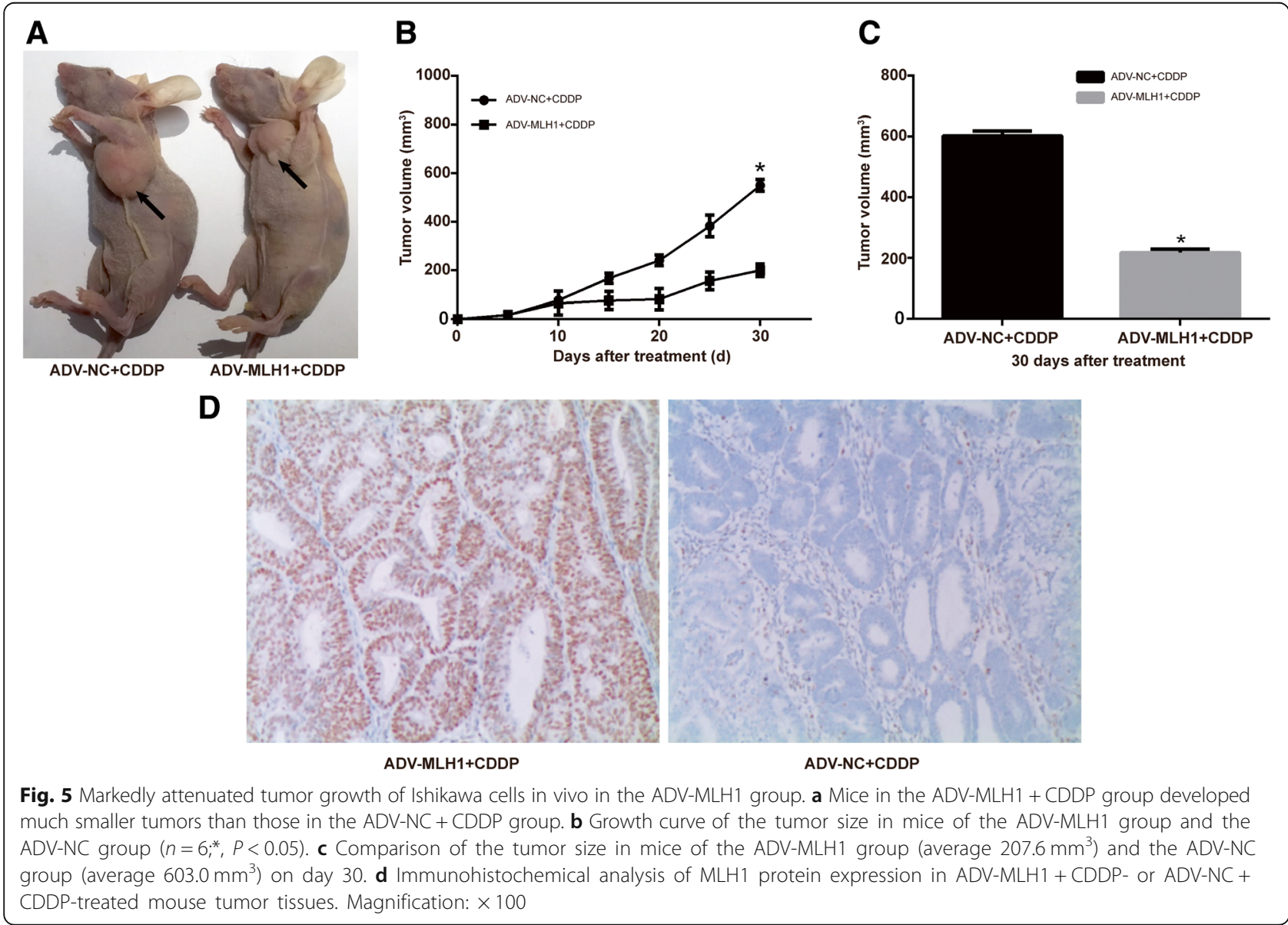


In this study, we detected reduced MLH1 protein and mRNA expression in Ishikawa cells compared with those in RL95-2 cells (Fig. 1a). Because Ishikawa cells are deficient in MLH1 and RL95-2 cells exhibit sufficient MLH1, Ishikawa and RL95-2 cells are ideal cell lines for determining the functional effects of MLH1. By overexpressing or downregulating MLH1 in Ishikawa or RL952 cells, respectively, we observed that MLH1 overexpression reduced cell proliferation properties (Fig. 2) and increased cell apoptosis (Fig. 3) in response to cisplatin, indicating that MLH1 plays an important role in the cisplatin sensitivity of human endometrial carcinoma cells. Consistent with our results, MLH1 has been reported to play an important role in cell apoptosis. Ding et al. [25] suggested that in the MMR-deficient ovarian cancer cell line A2780MNU1, MLH1 overexpression significantly reduced cell survival and proliferation, with a high percentage of cells displaying active caspase- 3 after drug treatment. Additionally, Ruzov et al. [26] showed that upregulation of MLH1 in HCT116 cells resulted in apoptosis during the onset of gastrulation in embryos. A similar effect was observed in a mouse xenograft model. In the present study, upregulation of ADV-MLH1 led to tumor growth arrest compared with tumors injected with ADV-NC (Fig. 5).

However, the mechanism by which MLH1 induces cell apoptosis in response to cisplatin is not fully understood. The c-Abl protein is a mediator of the MLH1-dependent cellular response to damage [27-29]. c-Abl, which exhibits tyrosine kinase activity, is a member of the nonreceptor tyrosine kinase Abelson superfamily, participating in the regulation of cell apoptosis and transformation, and its activation or mutation presents an important relationship with the malignant transformation of tumor cells. Therefore, we investigated whether c-Abl plays a critical role in cisplatin-induced cell apoptosis. In our study, overexpression of ADV-MLH1 activated the phosphorylation of c-Abl and caused a series of changes in downstream proteins (Fig. 4). Notably, phosphorylated c-Abl was robustly enhanced with prolonged cisplatin treatment. These results suggest that c-Abl phosphorylation moderates the apoptotic effects of cisplatin on endometrial carcinoma cells. Thus, c-Abl phosphorylation is important for apoptotic activation, and Hantschel suggested that phosphorylation occurs at tyrosine sites [30]. $\mathrm{Li}$ et al. [20] found that MLH1-overexpressing cells induced cell apoptosis in a p53-independent manner; however, when c-Abl transfectants were knocked down, MLH1-dependent apoptosis was prevented. Kim et al. [28] reported that the MEKK-1/MKK4/JNK/c-Jun pathway was triggered by MLH1 in response to the alkylator $\mathrm{N}$-methyl- $\mathrm{N}^{\prime}$-nitro- $\mathrm{N}^{\prime}$-nitrosoguanidine (MNNG). They also found that $\mathrm{c}-\mathrm{Abl}$ is required for the activation of this signaling cascade by MLH1. These authors observed a functional interacting complex between MLH1 and c-Abl in which MLH1 induced apoptosis, and this effect was dependent on the phosphorylation of c-Abl. Thus, evidence strongly suggests that phosphorylated c-Abl may be correlated with the tumor-suppressive effect of MLH1 in endometrial carcinoma cells. Regarding cisplatin sensitivity, these results further strengthen our understanding of MLH1 and indicate that MLH1 expression levels may be predictors of natural and acquired cisplatin resistance. Furthermore, from a clinical perspective, a decreased MLH1 repair capability is associated with resistance to chemotherapy drugs and a poor cancer prognosis [17].

In this study, we also constructed an adenovirus vector to be used for MLH1 overexpression. ADVs are doublestranded DNA viruses without an envelope. ADVs do not integrate into the host cell genome. Thus, the ADV vectors exhibit high safety and have been increasingly applied in clinical trials related to gene therapy. In our study, intratumoral injection of the ADV-MLH1 vector significantly suppressed tumor growth in vivo. This novel strategy provides a potential tool for gene therapy of endometrial cancer.

Our study had some limitations. Overexpression via ADV typically produces superphysiological levels of a protein that cannot recapitulate the physiological role of the protein. Thus, considerable work should be performed to investigate the mechanisms underlying MLH1 deficiency in endometrial carcinoma. The detailed mechanism is still not completely understood, and other MMR components (MSH2, PMS2, MSH6) may be functionally related to MLH1. All of these topics require further study.

\section{Conclusions}

In conclusion, our study indicated that MLH1 plays an important role in improving the cisplatin sensitization of endometrial carcinoma cells through activation of the MLH1/c-Abl signaling pathway in vitro. Using a mouse xenograft model, we demonstrated that Ishikawa cells infected with ADV-MLH1 displayed markedly attenuated tumor growth in vivo, further supporting a proapoptosis role of MLH1 in endometrial carcinoma cells. This newly identified mechanism helps provide a better understanding of carcinogenesis, and the ADV-MLH1 adenovirus vector may serve as a novel potential therapeutic treatment for human endometrial carcinoma.

\section{Additional files}

Additional file 1: Figure S1. Adenovirus vector map. (PDF $57 \mathrm{~kb}$ )
Additional file 2: Figure S2. Atlas of MLH1 Full Length Gene Sequencing.
(PDF $821 \mathrm{~kb})$
Additional file 3: Figure S3. Results of MLH1 Full Length Gene
Sequencing. (PDF $832 \mathrm{~kb})$
Additional file 4: Table S1. The information about the sequences.
$(\mathrm{XLS} 21 \mathrm{~kb})$




\section{Abbreviations}

ADV-MLH1: Adenovirus vector-MLH1; ADV-NC: Adenovirus vector-negative control; ARRIVE: Animal Research: Reporting of In Vivo Experiments; BCL-2: Bcell lymphoma-2; CCK-8: Cell counting kit-8; CDDP: Cis-dichlorodiammine platinum; DMSO: Dimethyl sulfoxide; ECL: Enhanced chemiluminescence; EDTA: Ethylenediaminetetraacetic acid; FACS: Fluorescence activated cell sorting; FBS: Fetal bovine serum; M-MLV: Moloney murine leukemia virus; MMR: Mismatch repair; MNNG: N-methyl-N'-nitro-N'-nitrosoguanidine; MOI: Multiplicity of infection; PARP: Poly ADP-ribose polymerase; PCR: Polymerase chain reaction; PI: Propidium iodide; qRT-PCR: Quantitative real time polymerase chain reaction; RPMI: Roswell park memorial institute; SDS: Sodium dodecyl sulfate

\section{Acknowledgments}

We wish to thank Professors Jinbo Feng and Zhenping Liu from the Gene Research Center of Qilu Hospital for technical assistance and for providing the cell lines.

\section{Funding}

This work was supported in part by funds from the National Natural Science Foundation of China (No. 81372809), the National Clinical Research Center for Gynecological Oncology (No. 2015BAl13B05), and the Department of Medical and Health Science Technology of Shandong Province (No. 2016WS0345). The funders had no role in study design, in data collection, analysis and interpretation, and in writing the manuscript.

\section{Availability of data and materials}

All data generated or analyzed during this study are included in this published article (and its Supplementary Information files). The datasets are available from the corresponding author upon reasonable request.

\section{Authors' contributions}

$Y L$ participated in the conception and design of the study, data analysis, interpretation and manuscript writing. SZ, YW and JP collected and assembled the data. FF analyzed the data. XY conceived the study and provided financial support. All authors read and approved the final manuscript.

\section{Ethics approval}

All experimental procedures were approved by the Laboratory Animal Ethics Committee of Qilu Hospital, Shandong University. The principles outlined in the ARRIVE (Animal Research: Reporting of In Vivo Experiments) guidelines and Basel declaration (including the $3 \mathrm{R}$ concept) were considered when planning experiments.

\section{Consent for publication}

Not applicable.

\section{Competing interests}

The authors declare that they have no competing interests.

\section{Publisher's Note}

Springer Nature remains neutral with regard to jurisdictional claims in published maps and institutional affiliations.

\section{Author details}

'Department of Obstetrics and Gynecology, Qilu Hospital of Shandong University, No. 107, Wenhua Xi Road, Jinan, Shandong 250012, People's Republic of China. ${ }^{2}$ Department of Obstetrics and Gynecology, Weihai Municipal Hospital, No. 70, Heping Road, WeiHai, Shandong 264200, People's Republic of China. ${ }^{3}$ Huaxi Clinical Medical College of Sichuan University, Jiang'an campus, Chengdu City, Sichuan 610207, People's Republic of China.

Received: 21 May 2018 Accepted: 13 December 2018

Published online: 29 December 2018

\section{References}

1. Stoffel E, Mukherjee B, Raymond VM, Tayob N, Kastrinos F, Sparr J, Wang F, Bandipalliam P, Syngal S, Gruber SB. Calculation of risk of colorectal and endometrial cancer among patients with lynch syndrome. Gastroenterology. 2009;137(5):1621-7.
2. Negrini S, Gorgoulis VG, Halazonetis TD. Genomic instability-an evolving hallmark of cancer. Nat Rev Mol Cell Biol. 2010;11(3):220-8.

3. Hanahan D, Weinberg RA. Hallmarks of cancer: the next generation. Cell. 2011;144:646-74.

4. Fink D, Nebel S, Aebi S, Zheng H, Cenni B, Nehmé A, Christen RD, Howell $\mathrm{SB}$. The role of DNA mismatch repair in platinum drug resistance. Cancer Res. 1996;56(21):4881-6.

5. Fink D, Aebi $S$, Howell SB. The role of DNA mismatch repair in drug resistance. Clin Cancer Res. 1998;4(1):1-6.

6. Mayer F, Gillis AJ, Dinjens W, Oosterhuis JW, Bokemeyer C, Looijenga LH. Microsatellite instability of germ cell tumors is associated with resistance to systemic treatment. Cancer Res. 2002;62(10):2758-60.

7. Velasco A, Corvalan A, Wistuba II, Riquelme E, Chuaqui R, Majerson A, Leach FS. Mismatch repair expression in testicular cancer predicts recurrence and survival. Int J Cancer. 2008;122(8):1774-7.

8. Kothandapani A, Dangeti VS, Brown AR, Banze LA, Wang XH, Sobol RW, Patrick SM. Novel role of base excision repair in mediating cisplatin cytotoxicity. J Biol Chem. 2011;286(16):14564-74.

9. Kothandapani A, Sawant A, Dangeti VS, Sobol RW, Patrick SM. Epistatic role of base excision repair and mismatch repair pathways in mediating cisplatin cytotoxicity. Nucleic Acids Res. 2013;41(15):7332-43.

10. Sawant A, Kothandapani A, Zhitkovich A, Sobol RW, Patrick SM. Role of mismatch repair proteins in the processing of cisplatin interstrand crosslinks. DNA Repair (Amst). 2015;35:126-36.

11. Schmittgen TD, Livak KJ. Analyzing real-time PCR data by the comparative C(T) method. Nat Protoc. 2008;3(6):1101-8.

12. Meyer C, Dzieran J, Liu Y, Schindler F, Munker S, Müller A, Coulouarn C, Dooley S. Distinct dedifferentiation processes affect caveolin-1 expression in hepatocytes. Cell Commun Signal. 2013;11(1):6.

13. Kruger NJ. The Bradford method for protein quantitation. In: methods in molecular biology. Clifton, NJ: Humana Press. 1994;32(32):9-15.

14. Li W, Lee MR, Choi E, Cho MY. Clinicopathologic significance of Survivin expression in relation to CD133 expression in surgically resected stage II or III colorectal Cancer. J Pathol Transl Med. 2016;51(1):17-23.

15. Lee JA, Spidlen J, Boyce K, Cai J, Crosbie N, Dalphin M, et al. MIFlowCyt: the minimum information about a flow cytometry experiment. Cytometry A. 2008;73(10):926-30.

16. Liu Z, Yao L, Tan B, Li L, Chen B. Detection of microRNA-200b may predict the inhibitory effect of gefitinib on non-small cell lung cancer and its potential mechanism. Oncol Lett. 2016;12(6):5349-55.

17. Clingen PH, Wu JY, Miller J, Mistry N, Chin F, Wynne P, Prise KM, Hartley JA. Histone $\mathrm{H} 2 \mathrm{AX}$ phosphorylation as a molecular pharmacological marker for DNA interstrand crosslink cancer chemotherapy. Biochem Pharmacol. 2008; 76(1):19-27

18. Jia J, Wang Z, Cai J, Zhang Y. PMS2 expression in epithelial ovarian cancer is posttranslationally regulated by Akt and essential for platinum-induced apoptosis. Tumor Biol. 2016;37(3):3059-69.

19. Vaisman A, Varchenko M, Umar A, Kunkel TA, Risinger Jl, Barrett JC, et al. The role of hMLH1, hMSH3, and hMSH6 defects in cisplatin and oxaliplatin resistance: correlation with replicative bypass of platinum-DNA adducts. Cancer Res. 1998:58(16):3579-85.

20. Li LS, Morales JC, Hwang A, Wagner MW, Boothman DA. DNA mismatch repair-dependent activation of c-Abl/ p73alpha/GADD45alpha-mediated apoptosis. J Biol Chem. 2008;283(31):21394-403.

21. Tomer G, Buermeyer AB, Nguyen MM, Liskay RM. Contribution of human mlh1 and pms2 ATPase activities to DNA mismatch repair. J Biol Chem. 2002;277(24):21801-9.

22. Lujan SA, Williams JS, Pursell ZF, Abdulovic-Cui AA, Clark AB, Nick McElhinny SA, Kunkel TA. Mismatch repair balances leading and lagging strand DNA replication fidelity. PLoS Genet. 2012;8(10):1003-6.

23. Martin LP, Hamilton TC, Schilder RJ. Platinum resistance: the role of DNA repair pathways. Clin Cancer Res. 2008;14(5):1291-5.

24. Papouli E, Cejka P, Jiricny J. Dependence of the cytotoxicity of DNA-damaging agents on the mismatch repair status of human cells. Cancer Res. 2004; 64(10):3391-4.

25. Ding X, Mohd AB, Huang Z, Baba T, Bernardini MQ, Lyerly HK, Berchuck A, Murphy SK, Buermeyer AB, Devi GR. MLH1 expression sensitises ovarian cancer cells to cell death mediated by XIAP inhibition. Br J Cancer. 2009:101(2):269-77.

26. Ruzov A, Shorning B, Mortusewicz O, Dunican DS, Leonhardt H. Meehan RR. MBD4 and MLH1 are required for apoptotic induction in XDNMT1-depleted embryos. Development. 2009;136(13):2277-86. 
27. Nehme A, Baskaran R, Nebel S, Fink D, Howell SB, Wang JY, Christen RD. Induction of JNK and C-Abl signalling by cisplatin and oxaliplatin in mismatch repair-proficient and -deficient cells. Br J Cancer. 1999; 79(7-8):1104-10.

28. Kim WJ, Rajasekaran B, Brown KD. MLH1- and ATM-dependent MAPK signaling is activated through $\mathrm{c}-\mathrm{Abl}$ in response to the alkylator $\mathrm{N}$-methyl-N'-nitro- $\mathrm{N}^{\prime}$ nitrosoguanidine. J Biol Chem. 2007;282(44):32021-31.

29. Wagner MW, Li LS, Morales JC, Galindo CL, Garner HR, Bornmann WG, Boothman DA. Role of c-Abl kinase in DNA mismatch repair-dependent G2 cell cycle checkpoint arrest responses. J Biol Chem. 2008;283(31):21382-93.

30. Hantschel O, Superti-Furga G. Regulation of the c-Abl and Bcr-Abl tyrosine kinases. Nat Rev Mol Cell Biol. 2004;5(1):33-44.

Ready to submit your research? Choose BMC and benefit from:

- fast, convenient online submission

- thorough peer review by experienced researchers in your field

- rapid publication on acceptance

- support for research data, including large and complex data types

- gold Open Access which fosters wider collaboration and increased citations

- maximum visibility for your research: over $100 \mathrm{M}$ website views per year

At BMC, research is always in progress.

Learn more biomedcentral.com/submissions 